\title{
CardioMPP: Uma Proposta para uma Assistência a Diagnóstico Avançada e Precisa
}

\author{
Allana Capistrano \\ de Oliveira \\ Instituto da \\ Computação \\ Universidade Federal \\ Fluminese \\ Niterói - Rio de Janeiro \\ allanaoliveira@id.uff.br
}

\author{
Luciana Cardoso de \\ Castro Salgado \\ Instituto da \\ Computação \\ Universidade Federal \\ Fluminese \\ Niterói - Rio de Janeiro \\ luciana@ic.uff.br
}

\author{
Flávio Luiz Seixas \\ Instituto da \\ Computação \\ Universidade Federal \\ Fluminese \\ Niterói - Rio de \\ Janeiro \\ fseixas@ic.uff.br
}

\author{
Mateus Kono \\ Instituto da \\ Computação \\ Universidade Federal \\ Fluminese \\ Niterói - Rio de \\ Janeiro \\ mateuskono@id.uff.br
}

\section{RESUMO}

A Cardiomiopatia Periparto é uma doença rara que tem sido disseminada ao longo do tempo em países subdesenvolvidos [4] resultando no aumento da mortalidade materna e na redução da taxa de nascidos vivos. Por ser pouco conhecida pelos médicos, o diagnóstico torna-se tardio ou inexistente. Assim sendo, o objetivo deste projeto é a criação de uma tecnologia da informação para prevenção, diagnóstico e assitência aos médicos especialistas das clínicas de Saúde da Família. A identificação de oportunidades para resolver o problemas segue o processo de design proposto pela Interação Humano-Computador (IHC): análise da situação atual, síntese de uma intervenção e avaliação da solução proposta. Através destas etapas, é possível definir o escopo do projeto para a criação de um aplicativo móvel capaz de otimizar o reconhecimento da doença e tratamento pelos médicos das clínicas de Saúde da Família.

\section{PALAVRAS-CHAVE}

Design de IHC, Aplicativo móvel, Cardiologia, Gestantes

\section{Introdução}

A Cardiomiopatia Periparto (CMPP) é uma doença negligenciada em seu tratamento por ser rara e carecer de conhecimento. A CMPP tem acometido em aumento da taxa de mortalidade materna e redução da taxa de nascidos vivos em países subdesenvolvidos [4]. Por meio deste fato, fora desenvolvido um aplicativo para apoiar os médicos no diagnóstico como forma de antecipar o tratamento e prevenção do desenvolvimento da doença.

Estudos têm sido realizados para definir como aplicativos utilizados na área da saúde devem ser desenvolvidos de forma a

\footnotetext{
Permission to reproduce or distribute, in whole or in part, material extracted from this work, verbatim, adapted or remixed, as well as the creation or production from the content of such work, is granted without fee for noncommercial use, provided that the original work is properly credited. IHC 2019 - TRILHA PÔSTERES E DEMONSTRAÇÕES, Outubro 21-25, 2019, Vitória, Brasil. In Anais Estendidos do XVIII Simpósio Brasileiro sobre Fatores Humanos em Sistemas Computacionais. Porto Alegre: SBC.

(C) 2019 by the author(s), in accordance with the terms of the Creative Commons Attribution-NonCommercial 4.0 International Public License (CC BY-NC 4.0).
}

respeitar a ética, adaptar-se à rotina médica, verificar possíveis dificuldades encontradas nas redes de saúde entre outros dilemas associados à inserção da tecnologia no ambiente médico [1]. $\mathrm{O}$ processo de design em IHC visa através de suas etapas assegurar que algumas destas dúvidas sejam sanadas ao viabilizar que as soluções desenvolvidas atendam a necessidade proposta de forma simples e objetiva [5].

O aplicativo CardioMPP foi desenvolvido para disseminar as informações sobre Cardiomiopatia Periparto e torná-la mais conhecida e acessível aos médicos. Para iniciar a elaboração de um projeto capaz de lidar com esta realidade e necessidade, conduzimos um processo de design em IHC, com as clínicas de Saúde da Família de Niterói (RJ) como referência de pesquisa e aquisição de informações. Após as primeiras etapas de design serem realizadas, o desenvolvimento de um aplicativo móvel que atende aos objetivos e dificuldades analisadas foi possível.

\section{Processo de Design da Interação}

O Processo de Design de IHC é fundamental para o desenvolvimento de um projeto de software com foco no usuário. O processo consiste em 3 etapas [5]: (a) análise da situação atual; (b) síntese de uma intervenção; (c) avaliação da solução proposta. Estas etapas funcionam como refinamento e melhoria das análises e requisições feitas a priori pelo médico pesquisador responsável.

A etapa de análise da situação atual (a) compreendeu as seguintes tarefas: (i) entrevista com médico pesquisador para aquisição de requisitos e necessidades que o projeto precisa atender; (ii) uma inspeção de aplicações similares existentes para obter um panorama do que é utilizado pelos médicos e quais são as facilidades aprendidas; (iii) criação de cenário de problema e cenários de interação para viabilizar uma análise das dificuldades, as quais podem ser encontradas numa rotina médica e organização do espaço de problema.

$\mathrm{Na}$ etapa da análise da situação atual (ii), a inspeção de aplicativos como WhiteBook ${ }^{1}$ e o Medscape $^{2}$ mostrou que tais

\footnotetext{
${ }^{1}$ Saiba mais sobre o WhiteBook: https://whitebook.pebmed.com.br/login

${ }^{2}$ Saiba mais sobre o Medscape: https://www.medscape.com/
} 
aplicações disponibilizam um conteúdo vasto relacionado à pesquisa médica. Em relação à doença alvo neste projeto, havia informações limitadas e gerais que não possibilitam o auxílio diagnóstico e o conhecimento completo da mesma. A etapa (iii) possibilitou enxergar que a rotina de um médico da Saúde da Família é de atendimentos diários de aproximadamente 30 gestantes. Este dado nos levou a definição de uma meta de design: tornar o conteúdo e uso do aplicativo útil e eficaz [6].

A partir da descrição do sistema e definição da árvore de decisão [2] com o protocolo de diagnóstico da doença, um protótipo não-funcional foi criado com o aplicativo $\mathrm{Marvel}^{3}$, como síntese de uma intervenção para validação das informações adquiridas (Figura 1). A elaboração do protótipo possibilitou uma avaliação feita por meio de observação e entrevista com um médico especialista da clínica de Saúde da Família. [5] mostra que IHC preocupa-se em diferenciar os stakeholders para garantir que o projeto atende de modo completo a ambos. Por isso, nesta etapa verificamos dificuldades que poderiam ser encontradas pelos médicos ao utilizar um aplicativo, garantindo levantamento de melhorias e adaptações a serem feitas na aplicação. Além disso, pode-se perceber a necessidade de uma aplicação, a qual funcione em modo offline, pois não são todas as clínicas que possuem uma rede local favorável. Outros detalhes foram analisados como forma de garantir uma melhor interação do médico com o aplicativo [3], como, a otimização da árvore de decisão utilizada na qualidade de fluxo e a preferência pelo uso de um aplicativo em modo de consulta ao invés de modo de cadastro.
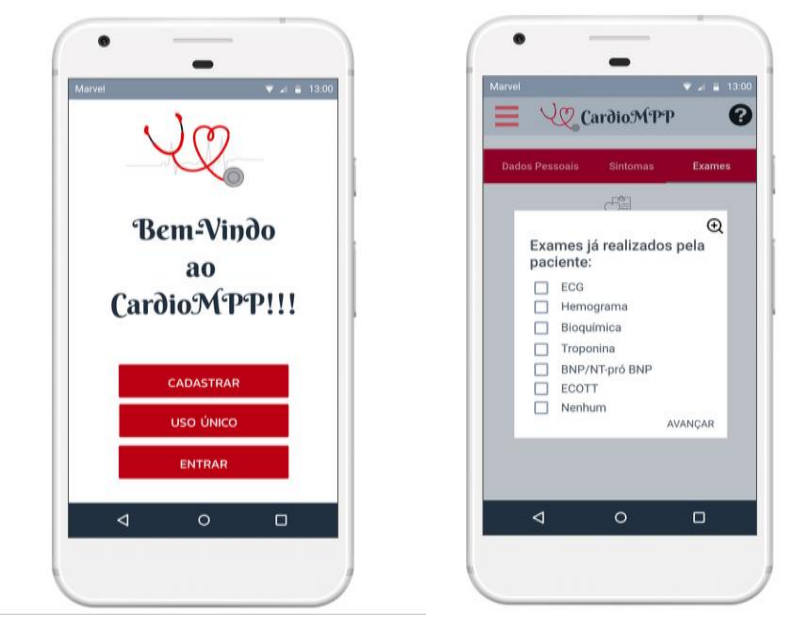

Figura 1: a. Tela inicial do protótipo. b. Exemplo de tela de decisão ao longo do diagnóstico.

\section{Projeto do Software}

Como descrito em [5], a colaboração entre as áreas de IHC e Engenharia de Software são imprescindíveis para a elaboração de um sistema que seja adequado em sua construção, onde a Engenharia de Software atua e uso, área de IHC. Sendo assim, após a primeira iteração das etapas do processo de design ter sido efetuada, o desenvolvimento da aplicação iniciou-se. A Engenharia do Software do aplicativo CardioMPP consistiu na adaptação do Scrum com a definição das metas e responsabilidades dos alunos, que participam do projeto, em uma reunião chamada planning. Além disso, também foi estipulado um tempo em que essas metas devem ser alcançadas (prazo da Sprint), que varia de acordo com as responsabilidades nas matérias que estão sendo cursadas.

O desenvolvimento do produto mínimo viável foi concluído. Com isso o aplicativo já contempla as funcionalidades de login, cadastro, recuperação de senha por e-mail e realização da consulta apoiada pela árvore de decisão [2]. Através de entrevistas e treinamentos realizados com os médicos identificamos que a aplicação móvel facilitará a rotina diária e potencialmente aumentará o entendimento dos médicos sobre o fluxo necessário de seguir para um diagnóstico antecipado da doença. A próxima etapa do projeto prevê o teste de usabilidade e atualização nas plataformas Apple Store e Google Play.

\section{Conclusão}

A rotina de uma clínica médica é muito intensa e, com isso, a elaboração de tecnologias que auxiliem a busca por diagnósticos eficientes de forma imediata é cada vez mais comum. Neste projeto vimos os benefícios da utilização de métodos de IHC desde a fase inicial de um projeto, pois além do entendimento do problema em profundidade, evitou retrabalhado na fase de implementação propriamente dita. A metodologia aplicada explorou alternativas de soluções de forma antecipada. Trabalhos futuros incluem a disponibilização da tecnologia nas clínicas de Saúde e o monitoramento do impacto na identificação da doença e na redução da mortalidade materna.

\section{AGRADECIMENTOS}

Agradecemos a Proppi e ao CNPQ, pelo apoio e fomento à pesquisa, e aos profissionais de saúde participantes do projeto e voluntários que fizeram deste projeto possível.

\section{REFERÊNCIAS}

[1] Blandford, A., Berndt, E., Catchpole, K., Furniss, D., Mayer, A., Mentis, H., O'Kane, A., Owen, T., Rajkome, A. \& Randell, R. (in press). Experiencing Interactive Healthcare Technologies: embracing 'the wild' on its own terms. Invited submission to ToCHI's special issue on 'The Turn to the Wild'.

[2] Circulation. Peripartum Cardiomyopathy Zolt Arany, MD, PhD; Uri Elkayam, MD 2016;133:1397-1409. DOI: 10.1161/ CIRCULATIONAHA.115.020491.

[3] Creswell, J. W. (2009). Research Design: Qualitative, Quantitative, and Mixed Methods Approaches. 3rd Edition. Los Angeles: Sage Publications.

[4] Desai D, Moodley J, Naidoo D. (1995). Peripartum cardiomyopathy: experiences at King Edward VIII Hospital, Durban, South Africa and a review of the literature. Trop Doct; 25:118-123.

[5] S. Diniz Barbosa, J.; B. Santana da Silva. (2010). Interação HumanoComputador. [S.1.]: Elsevier Editora Ltda.

[6] Stuart Reeves. 2011. Designing Interfaces in Public Settings: Understanding the Role of the Spectator in Human-Computer Interaction (1st ed.). Springer Publishing Company, Incorporated.

\footnotetext{
${ }^{3}$ Saiba mais sobre o Marvel: https://marvelapp.com/
} 